\title{
Improved spatial separation of neutral molecules
}

Jens S. Kienitz, Karol Długołęcki, Sebastian Trippel, and Jochen Küpper

Citation: The Journal of Chemical Physics 147, 024304 (2017); doi: 10.1063/1.4991479

View online: http://dx.doi.org/10.1063/1.4991479

View Table of Contents: http://aip.scitation.org/toc/jcp/147/2

Published by the American Institute of Physics

\section{Articles you may be interested in}

Accurate spectroscopic characterization of the $\mathrm{HOC}(\mathrm{O}) \mathrm{O}$ radical: A route toward its experimental identification

The Journal of Chemical Physics 147, 024302 (2017); 10.1063/1.4990437

A molecular dynamics study of nuclear quantum effect on diffusivity of hydrogen molecule

The Journal of Chemical Physics 147, 024501 (2017); 10.1063/1.4991732

Representations in neural network based empirical potentials

The Journal of Chemical Physics 147, 024104 (2017); 10.1063/1.4990503

Coupled cluster valence bond theory for open-shell systems with application to very long range strong correlation in a polycarbene dimer

The Journal of Chemical Physics 147, 024107 (2017); 10.1063/1.4991797

Unveiling the nature of post-linear response Z-vector method for time-dependent density functional theory The Journal of Chemical Physics 147, 024108 (2017); 10.1063/1.4991561

Perspective: Outstanding theoretical questions in polymer-nanoparticle hybrids

The Journal of Chemical Physics 147, 020901 (2017); 10.1063/1.4990501

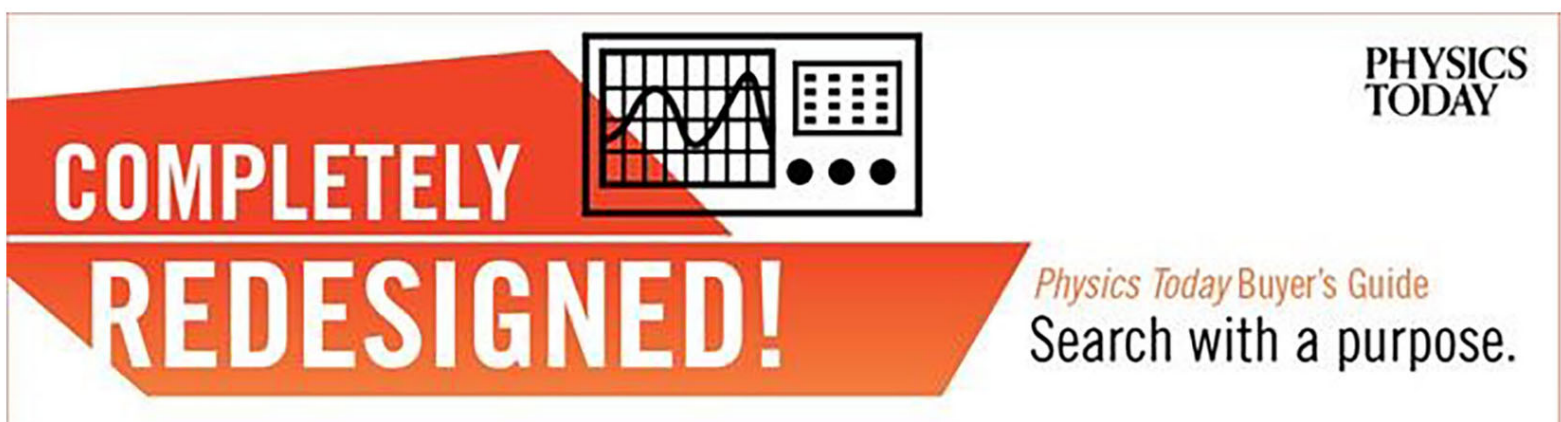




\title{
Improved spatial separation of neutral molecules
}

\author{
Jens S. Kienitz, ${ }^{1,2}$ Karol Długołęcki, ${ }^{1}$ Sebastian Trippel, ${ }^{1,2, a)}$ and Jochen Küpper ${ }^{1,2,3, b)}$ \\ ${ }^{1}$ Center for Free-Electron Laser Science, Deutsches Elektronen-Synchrotron DESY, Notkestrasse 85, \\ 22607 Hamburg, Germany \\ ${ }^{2}$ The Hamburg Center for Ultrafast Imaging, University of Hamburg, Luruper Chaussee 149, \\ 22761 Hamburg, Germany \\ ${ }^{3}$ Department of Physics, University of Hamburg, Luruper Chaussee 149, 22761 Hamburg, Germany
}

(Received 28 April 2017; accepted 21 June 2017; published online 13 July 2017)

\begin{abstract}
We have developed and experimentally demonstrated an improved electrostatic deflector for the spatial separation of molecules according to their dipole-moment-to-mass ratio. The device features a very open structure that allows for significantly stronger electric fields as well as for stronger deflection without molecules crashing into the device itself. We have demonstrated its performance using the prototypical carbonyl sulfide molecule and we discuss opportunities regarding improved quantumstate-selectivity for complex molecules and the deflection of unpolar molecules. Published by AIP Publishing. [http://dx.doi.org/10.1063/1.4991479]
\end{abstract}

\section{INTRODUCTION}

The history of the deflection of neutral atoms and molecules in vacuum began in 1922 with the famous SternGerlach experiment, ${ }^{1-3}$ which exploited the Zeeman effect of silver atoms and the corresponding force in an inhomogeneous magnetic field. Just a few years later, the deflection of molecules by an inhomogeneous electric field was demonstrated. ${ }^{4,5}$ Various deflector shapes and techniques have evolved to optimize the process, ${ }^{6-9}$ and an overview of various early experimentally employed deflection-field geometries was already presented in Ramsey's textbook on molecular beams. ${ }^{10}$ Modern experiments typically employed geometries approximating two-wire fields. ${ }^{6,7}$ The technique allowed for a wide range of applications, such as the separation of molecules in individual rotational states, ${ }^{11-13}$ of conformers, ${ }^{14-16}$ and of specific molecular clusters. ${ }^{17}$ Additionally, the deflector enables the separation of polar molecules from the seed gas, which is relevant, for instance, for gas-phase diffraction experiments. ${ }^{18-21}$

Alternatively, eigenstates of small polar neutral molecules can be separated using switched electric or magnetic fields. ${ }^{12,22-28}$ Large molecular ions were separated according to their shape using ion-mobility techniques. ${ }^{29,30}$ Furthermore, laser fields have been used to deflect and decelerate neutral molecules, ${ }^{31-34}$ which also works for non-polar molecules. Laser-based electric deflection has demonstrated state-separability, ${ }^{34}$ but is limited by very small volumes, typically only manipulating a small subset of the molecular beam. The polarizability interaction was also used in the deceleration of hydrogen molecules in Rydberg states ${ }^{35}$ and in weak-deflection polarizability measurements. ${ }^{6,36}$ In addition, inhomogeneous ac- and dc-electric fields are

\footnotetext{
a) Electronic mail: sebastian.trippel@cfel.de

b) Electronic mail: jochen.kuepper@cfel.de. URL: https://www.controlledmolecule-imaging.org.
}

predicted to manipulate the average deflection angle and its distribution when combined with strongly aligned molecular samples. ${ }^{37,38}$

We have developed a Stark deflector for increased separation of cold molecular species in a beam that utilizes an improved geometry and supports stronger fields. The geometry is similar to previously proposed ${ }^{8,39}$ and utilized ${ }^{40}$ deflectors but is unique in its applicability for the separation of molecular species in dense molecular beams. ${ }^{9}$ Here, we experimentally demonstrate its performance for the separation of the eigenstates of carbonyl sulfide (OCS). We have characterized the deflection power through column-density measurements and the separation of eigenstates through statespecific mixed-field orientation measurements. ${ }^{41,42}$ Moreover, we have performed simulations on the deflection of non-polar molecules and discuss the feasibility of such experiments.

\section{THEORY}

\section{A. Stark effect}

In first order, the interaction of a molecule with a dc electric field, the Stark effect, is governed by the so-called permanent dipole moment $\boldsymbol{\mu},{ }^{9,43}$

$$
W^{(1)}=-\boldsymbol{\mu} \cdot \boldsymbol{\epsilon}=-\mu \epsilon\langle\cos \theta\rangle,
$$

and by the polarizability tensor, $\alpha$, or induced-dipole, $\boldsymbol{\mu}_{\text {ind }}$, interaction $^{43}$

$$
W^{(2)}=\frac{1}{2} \boldsymbol{\epsilon} \boldsymbol{\alpha} \epsilon=-\mu_{\text {ind }} \cdot \boldsymbol{\epsilon} .
$$

The effective, space-fixed, dipole moment $\mu_{\text {eff }}=-\partial W / \partial \epsilon$, the derivative of the energy $W=W^{(1)}+W^{(2)}$ with respect to the electric field, directly describes the interaction strength of the molecule with the field and the corresponding force in 
an inhomogeneous electric field, ${ }^{9,27}$

$$
\begin{aligned}
& W=\mu_{\text {eff }} \epsilon, \\
& \boldsymbol{F}=\mu_{\text {eff }} \nabla \epsilon .
\end{aligned}
$$

Stark effect calculations were performed using a modified version of the CMIstark program package ${ }^{44}$ with added polarizability matrix elements according to (2).$^{43}$ The resulting energies and effective dipole moments of the lowest-energy states of OCS are shown in Fig. 1. For these field strengths, the effect of the polarizability is below $1 \%$ and is not visible in the data in Fig. 1.

\section{B. Deflector design}

The design of the deflector was governed by the need to accept dense cold molecular beams, such as generated in high-pressure expansions from Even-Lavie valves. ${ }^{9,45}$ These beams have internal temperatures below $1 \mathrm{~K}$, but in order to avoid beam heating, they require large, multi-millimeter diameter skimmers and correspondingly large mechanical apertures for the deflector. Furthermore, strong electric fields and field gradients allow for strong deflection and separation. The fields should be strong enough for the molecules to be in the pendular regime ${ }^{9,27}$ and the field gradient should be fairly constant over the cross section covered by the molecular beam. In high-voltage breakdown tests, we were able to generate static electric fields up to $750 \mathrm{kV} / \mathrm{cm}$ before breakdowns occurred; however, these breakdown voltages depend on the actual vacuum gap ${ }^{46}$ and, thus, ask for small structures, while constant gradients again require large mechanical structures.

Here, we settled on a mechanical aperture of the deflector larger than $1.5 \mathrm{~mm}$ with a $1.5 \mathrm{~mm}$-diameter aperture conical skimmer before the device far enough from the valve to avoid choking and heating. We decided to limit applied voltages to $\pm 30 \mathrm{kV}$ to allow for corresponding feedthroughs.

The experimental realization of fields that satisfy such conditions was discussed before. ${ }^{8,10,27,47}$ We express the shape of the deflector by an equipotential line of a two-dimensional multipole expansion of the electric potential, $, 27,48$

$\Phi(x, y)=\Phi_{0}\left[\sum_{n=1}^{\infty} \frac{a_{n}}{n}\left(\frac{r}{r_{0}}\right)^{n} \cos (n \theta)+\sum_{n=1}^{\infty} \frac{b_{n}}{n}\left(\frac{r}{r_{0}}\right)^{n} \sin (n \theta)\right]$
TABLE I. Parameters according to (5) for the two deflector geometries discussed in the text. The mechanical scale in our simulations was fixed at $r_{0}$ $=3.3 \mathrm{~mm}$.

\begin{tabular}{lcr}
\hline \hline & " $a$-type" & " $b$-type" \\
\hline$a_{1} / b_{1}$ & 0.50 & 1.94 \\
$a_{2} / b_{2}$ & 0.49 & -4.80 \\
$a_{3} / b_{3}$ & 0.42 & 1.00 \\
\hline \hline
\end{tabular}

with $r=\sqrt{x^{2}+y^{2}}$ and $\theta=\tan ^{-1}(y / x) ; r_{0}$ and $\Phi_{0}$ are scaling factors of the geometric size and the potential values, respectively. To obtain deflection in the $x$ direction, either $a_{n}$ or $b_{n}$ can be set to zero, which results in a so-called " $a$-type" or " $b$-type" deflector according to the non-zero coefficients, respectively. The influence of these coefficients was discussed extensively elsewhere. . $^{87,47}$ We use the $b$-type shape to allow for an open mechanical structure along the deflection direction. Moreover, we allow for arbitrarily complex numerically defined electrode shapes to most closely follow the equipotential lines. The parameters obtained from a numerical optimization of the $b_{n}(n=1,2,3)$ coefficients $^{49}$ as well as the parameters of the previously used $a$-type deflector are specified in Table I. Cross sections of the corresponding fields, equipotential lines, and electrode geometries are depicted in Fig. 2. The applied voltages correspond to a maximum field strength of $363 \mathrm{kV} / \mathrm{cm}$, which corresponds to the experiments discussed below.

Actual three-dimensional structures were obtained by extruding the calculated 2D cross sections and rounding the ends with a radius of $2 \mathrm{~mm}$. For the $b$-type deflector, the equipotential lines were reproduced for $\phi=1$ and $x>-2.73 \mathrm{~mm}$ and mirrored to $x<-2.73 \mathrm{~mm}$. The resulting cusp was removed by approximating this geometry by a tenth-order even polynomial Taylor expansion over the range $-5.84 \mathrm{~mm}<x$ $<0.37 \mathrm{~mm}$. This symmetry of the deflector allows the deflection of molecules in both the directions depending on the relative positioning of the deflector and beam.

\section{EXPERIMENTAL SETUP}

The experimental setup is sketched in Fig. 3. A mixture of 500 ppm OCS seeded in 100 bar of helium was expanded into vacuum through an Even-Lavie valve ${ }^{50}$ at a
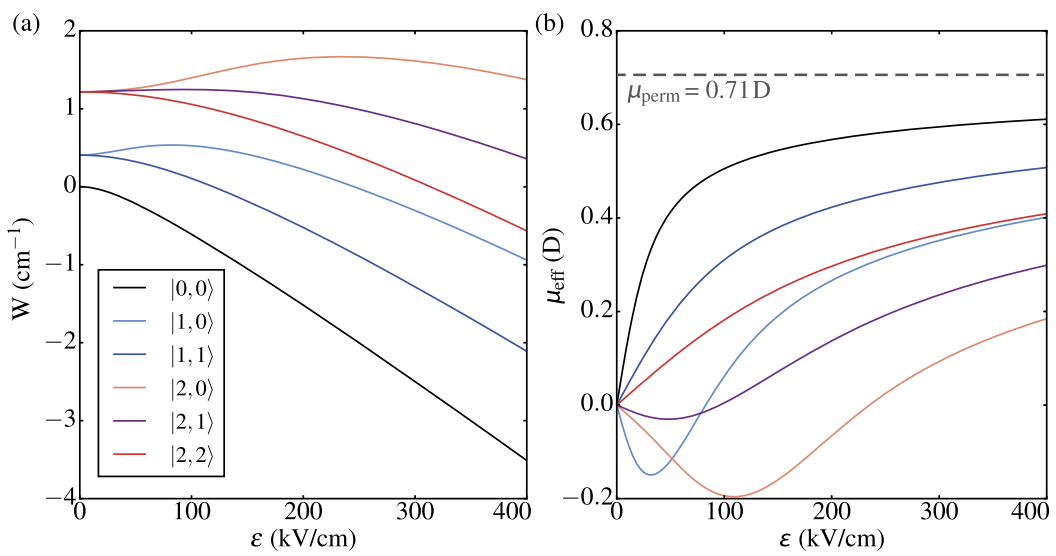

FIG. 1. (a) Energies of the lowest-energy states of OCS in a static electric field and (b) the corresponding effective dipole moments. The gray line depicts the strong-field limit of the permanent dipole moment. 

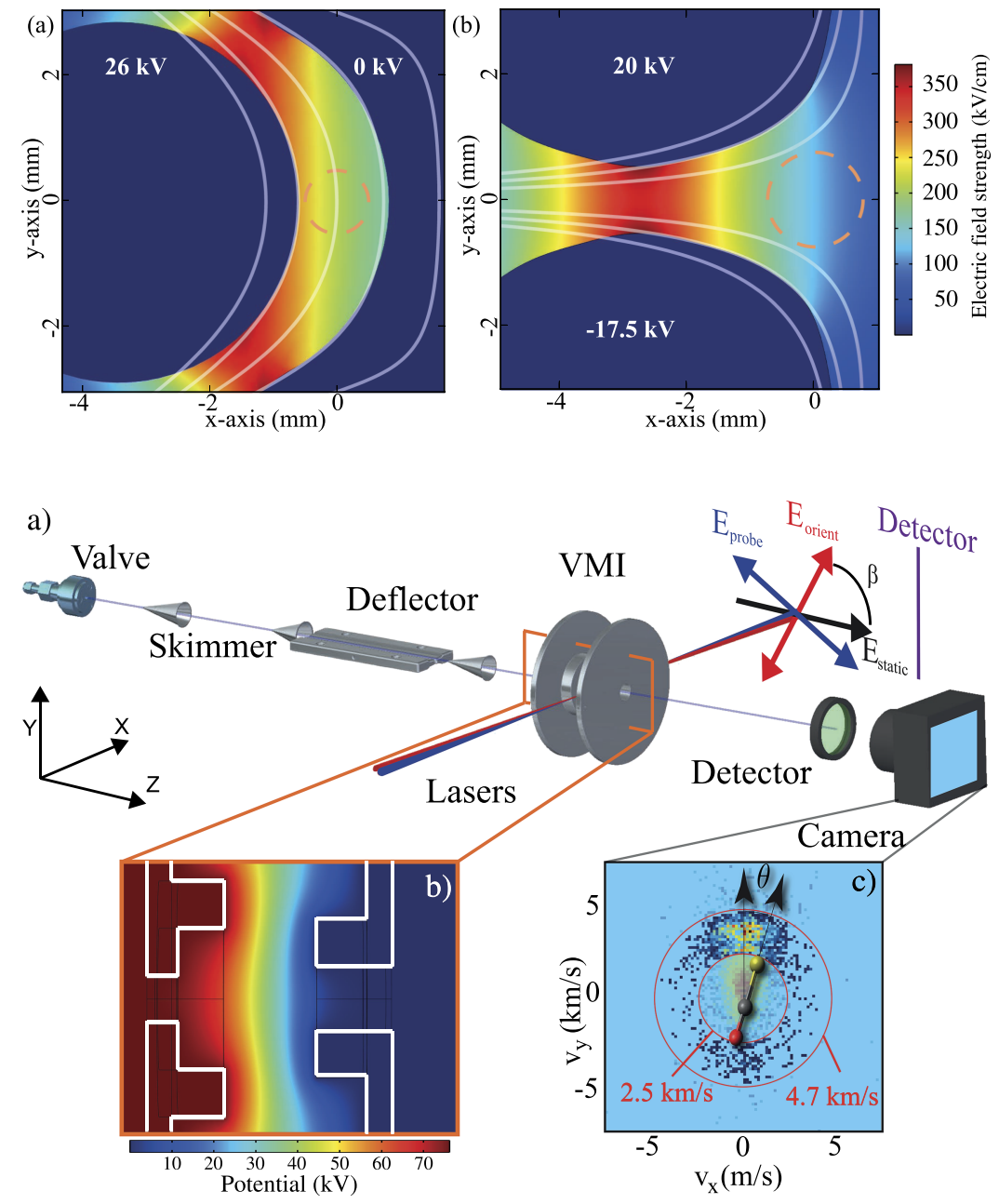

FIG. 2. Shape and electric fields of the (left) $a$-type and (right) $b$-type deflector geometries. The numerically calculated electric field strengths for the actual electrode geometries are depicted in color coding and white lines depict the equipotential lines according to (5), the $a_{i} / b_{i}$ coefficients specified in Table I, for $\phi / \phi_{0}$ ratios of (a) $-20,-10,0,10,20$ (left to right) and (b) 0.6, 1.0, and 1.4 (inside to outside). The dashed orange circles represent the utilized skimmers and mark the position and size of the incoming molecular beam. In both cases, molecules in high-field-seeking states are deflected to the left; in the experiments discussed below, the deflector is rotated counterclockwise by $90^{\circ}$.
FIG. 3. (a) Experimental setup with the definition of the axis system. The angle between the static electric field and the polarization vector of the control laser pulse is defined by $\beta$. (b) Electric field in the velocity-map imaging spectrometer with white lines depicting the electrode surfaces. (c) Velocity-map image of $\mathrm{S}^{+}$ions from OCS with red circles depicting the velocities defining the region of interest for the data analysis. See text for details. repetition rate of $250 \mathrm{~Hz}$. Two conical skimmers $(\varnothing 4 \mathrm{~mm}$ and $\varnothing 1.5 \mathrm{~mm}$ ) were placed $10.7 \mathrm{~cm}$ and $21.6 \mathrm{~cm}$ downstream from the nozzle, respectively. The Stark deflector was located $4.4 \mathrm{~cm}$ behind the tip of the second skimmer. The deflector was wire-eroded and electro-polished. After careful high-voltage conditioning of the electrodes, a voltage difference of $38 \mathrm{kV}$ was applied, which corresponds to the fields shown in Fig. 2(b). A third, transversely adjustable skimmer ( $\varnothing 1.5 \mathrm{~mm}$ ) was placed $4.7 \mathrm{~cm}$ downstream of the end of the deflector.

Two laser pulses, both provided by an amplified femtosecond laser system with a central wavelength of $800 \mathrm{~nm}$ and propagating along a common line, were used to orient and probe the molecules. ${ }^{51}$ The temporal profile of the control laser pulse had a sawtooth shape with a slow rising edge $(680 \mathrm{ps}$, $2.5 \%-97.5 \%)$ and a fast falling edge (190 ps). The spatial intensity profile in the interaction volume had widths of $\sigma=16 \mu \mathrm{m}$ and $\sigma^{\prime}=21 \mu \mathrm{m}$ along the two principal axes of the profile. The principal axes were rotated by $20^{\circ}$ from the $Y$ axis. The peak intensity of the control laser was $\mathrm{I}_{\text {control }} \approx 5 \times 10^{11} \mathrm{~W} / \mathrm{cm}^{2}$. Short pulses of a $30 \mathrm{fs}$ laser focused to $\sigma_{1}=\sigma_{2}=20 \mu \mathrm{m}$ and peak intensities of $\mathrm{I}_{\text {probe }} \approx 10^{14} \mathrm{~W} / \mathrm{cm}^{2}$ were used to multiple ionize the molecules to induce Coulomb explosion. The relative delay between both laser pulses was controlled with a motorized delay stage. Both laser pulses were linearly polarized perpendicular with respect to each other. The control laser polarization is at a fixed angle $\beta=45^{\circ}$ with respect to the $Z$-axis.

A high-voltage velocity-map-imaging spectrometer was used to record the momenta of $\mathrm{S}^{+}$ions as a signature of the molecules' directions at the time of ionization. ${ }^{42}$ Vertical molecular beam profiles, with and without voltages applied to the deflector, were recorded by moving the skimmer and the laser focus through the molecular beam and recording the integrated number of $\mathrm{S}^{+}$ions at each position. The envelope of all events over all skimmer positions yields the overall beam profile. Furthermore, we derived the degree of orientation across the molecular beam with the control laser pulses applied from the angular distribution of $\mathrm{S}^{+}$ions in the region of interest $\left(2500 \mathrm{~m} / \mathrm{s}<v_{x y}<4700 \mathrm{~m} / \mathrm{s}\right)$ shown in Fig. 3(c).

\section{RESULTS}

\section{A. Deflection of OCS}

Figure 4(a) shows the normalized measured (triangles) vertical density profiles of the deflected molecular beam for selected skimmer positions at $-2.75 \mathrm{~mm}$ (red), $-1.75 \mathrm{~mm}$ (yellow), $-0.75 \mathrm{~mm}$ (green), $0.25 \mathrm{~mm}$ (cyan), and $1.25 \mathrm{~mm}$ (purple), respectively. A strong dependence of the density profile on the position of the third skimmer is observed. Corresponding simulated ${ }^{7}$ beam-density 


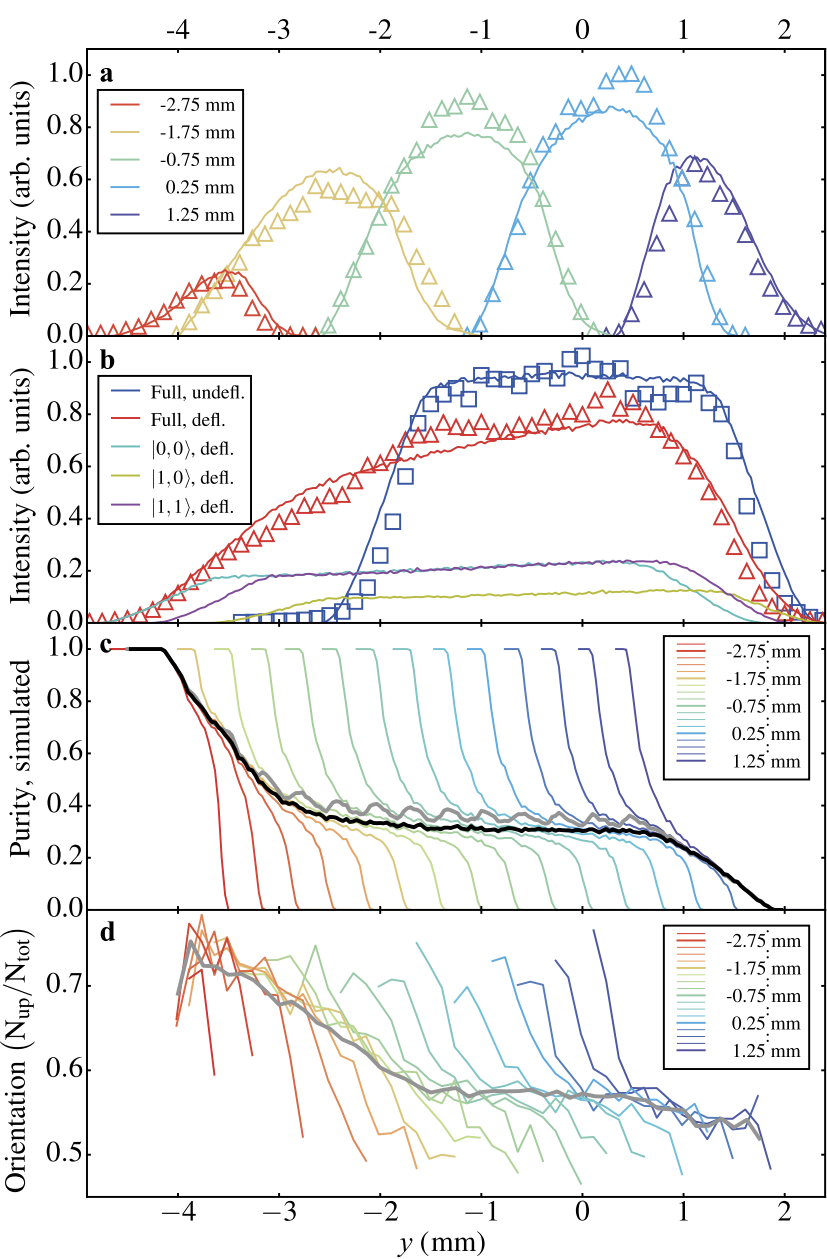

FIG. 4. (a) Experimental vertical density profiles for selected skimmer positions. (b) Experimental vertical density envelope profiles of the undeflected (blue squares) and deflected (red triangles) molecular beams. Simulated envelope profiles are shown as solid blue and red lines. Profiles of the individual rotational states $|0,0\rangle,|1,0\rangle$, and $|1,1\rangle$ are shown as cyan, yellow, and magenta lines, respectively. (c) Simulated purity of the $|0,0\rangle$ state for various skimmer positions. (d) Experimental degree of orientation for various skimmer positions. See text for details.

profiles are shown as solid lines. Each simulated profile fits the corresponding measured profile assuming a rotational temperature of $0.8 \mathrm{~K}$.

Figure 4(b) shows the normalized measured vertical density profiles of the undeflected (blue squares) and deflected (red triangles) molecular beams. The profiles are obtained from the envelope of 18 measured single profiles recorded at specific skimmer positions between $-3.0 \mathrm{~mm}$ and $1.25 \mathrm{~mm}$ with a relative step size of $0.25 \mathrm{~mm}$. All molecules are deflected downwards when $20 \mathrm{kV}$ and $-17.5 \mathrm{kV}$ are applied to the deflector electrodes, as all quantum states are high-field seeking at the electric field strengths experienced inside the deflector. The corresponding simulated beam-density profiles are shown as solid blue and red lines. The profiles of the individual rotational states $|0,0\rangle,|1,0\rangle$, and $|1,1\rangle$, which are the states that are deflected the most, are shown as cyan, yellow, and magenta lines, respectively. Again, the simulated profiles fit best to a rotational temperature of $0.8 \mathrm{~K}$. For positions $y<-3.5 \mathrm{~mm}$, practically only the $|0,0\rangle$ and $|1,1\rangle$ states are populated. According to the simulations, a pure ground state population is observed for positions below $y=-4.2 \mathrm{~mm}$.
The simulated purity $p_{i}=N_{|0,0\rangle_{i}} / N_{i}$ of the $|0,0\rangle$ state is shown for each specific skimmer position $i$ as colored lines in Fig. 4(c). The number of molecules in the absolute ground state and the total number of molecules are denoted by $N_{|0,0\rangle_{i}}$ and $N_{i}$, respectively. The purity changes continuously from 0 to 1 with decreasing laser focus positions for all skimmer positions. The simulated purity of the molecular beam without the last skimmer is shown as a black line. A gray line represents the weighted mean of the purity obtained for each position $p(y)=\sum p_{i}(y) N_{i}(y) / \sum N_{i}(y)$.

Figure 4(d) shows the measured degree of orientation $o(y)$ $=N_{\text {up }} / N_{\text {down }}$ across the deflected molecular beam for every skimmer position $i$ as colored lines. As previously shown, ${ }^{42,52}$ the degree of orientation is a good measure of the purity. The degree of orientation increased for decreasing vertical positions for each skimmer position $i$. The gray line shows the weighed mean of the degree of orientation obtained from the individual skimmer positions $o(y)=\sum o_{i}(y) N_{i}(y) / \sum N_{i}(y)$. An average degree of orientation of 0.58 was observed at positions between $y=-1.5$ and $y=1 \mathrm{~mm}$ where all states are present. The degree of orientation decreases to 0.54 for increasing $y$ with $y>1 \mathrm{~mm}$. In that region, only higher states $(J>0)$ are present. The degree of orientation rises from 0.58 to 0.75 in the region between $y=-1.5 \mathrm{~mm}$ and $y=-4 \mathrm{~mm}$. Only states with $J<2$ are present in that region. The ground state purity and, therefore, the degree of orientation are increased for decreasing vertical positions $y$.

\section{B. Simulated deflection of unpolar molecules}

To study further the performance of the new deflector, we have computationally investigated its applicability to the deflection and separation of non-polar neutral molecules. The Stark energy curves and effective dipole moments of the $J$ $=0,1$ states of the hydrogen isotopologues $\mathrm{H}_{2}, \mathrm{HD}$, and $\mathrm{D}_{2}$ are shown in Figs. 5(a) and 5(b). In these calculations, rotational and centrifugal distortion-constants were taken from Refs. 53 and 54 and polarizability anisotropies as specified in Table II. All Stark energies decrease as a function of electric field strength $\epsilon$ and, correspondingly, all effective dipole moments $\mu_{\mathrm{eff}}$ are positive and, therefore, attracted to regions of a stronger electric field. A linear increase of the effective dipole moments $\mu_{\text {eff }}$ as a function of electric field strength $\epsilon$ was observed for all quantum states and isotopologues. The largest differences in the slope of $\mu_{\text {eff }}$ were observed between different initial rotational states. Only small differences were found between the three isotopologues in a given specific rotational quantum state. The simulated deflection profiles for a molecular beam with a rotational temperature of $T_{r}=1 \mathrm{~K}$ are shown in Fig. 5(c). The speed of the simulated molecular beam was $385 \mathrm{~m} / \mathrm{s}$, which was demonstrated for molecular beams seeded in krypton. ${ }^{59}$ The black line depicts the overall profile for the undeflected beam, without applying any field. It has an identical shape for all isotopologues. The colored lines are the deflection profiles of the individual isotopologues for an applied maximum field strength of $363 \mathrm{kV} / \mathrm{cm}$. All hydrogen isotopologues were deflected significantly in the simulation. The largest deflection was observed for hydrogen which has the smallest mass of the three isotopologues. 

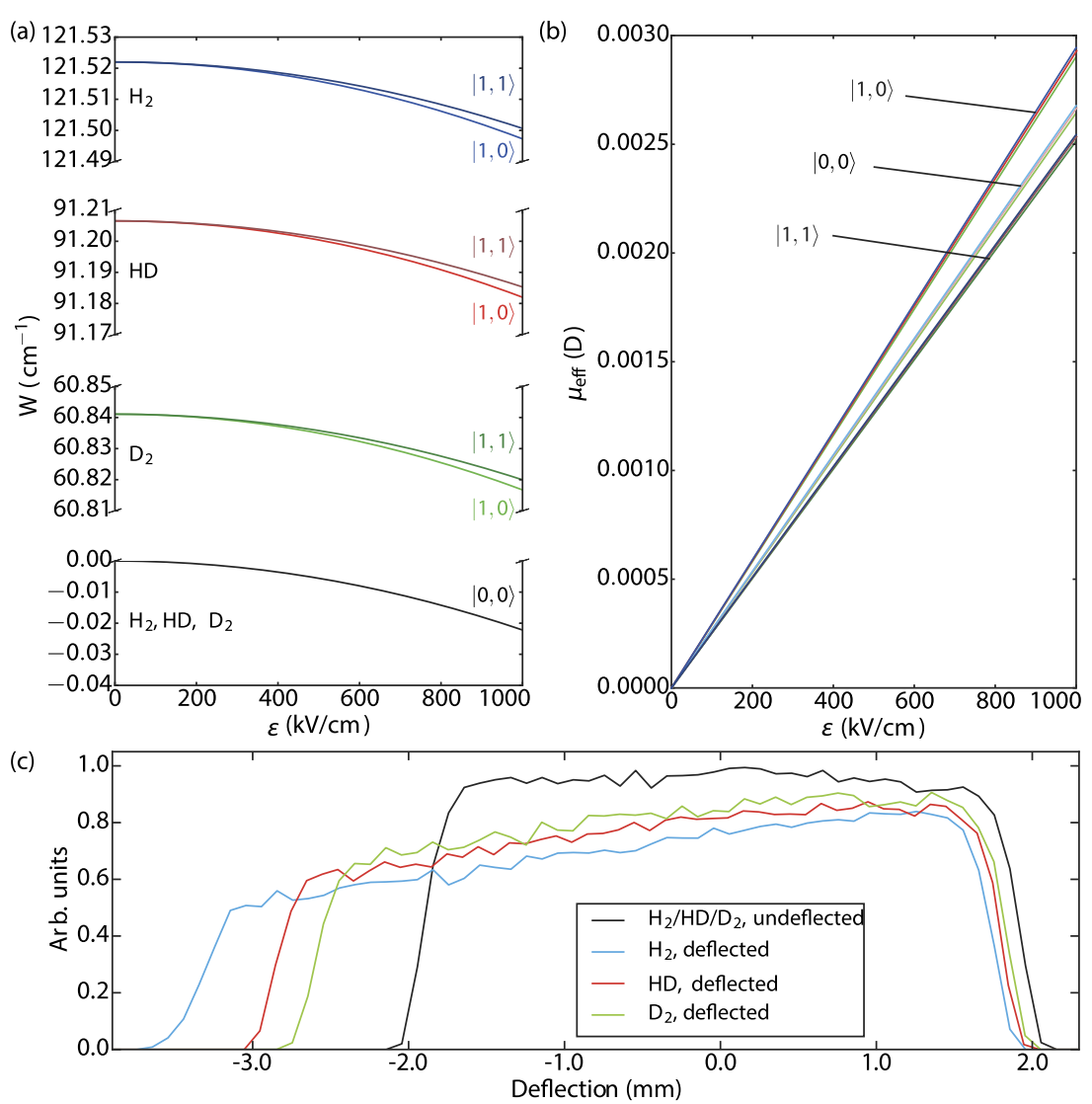

FIG. 5. (a) Stark energies and (b) effective dipole moments of the $J=0,1$ states of hydrogen molecule isotopologues. Both plots use the same color coding for the rotational states. (c) Vertical molecular beam profiles without deflection (black) and with deflection for $\mathrm{H}_{2}$ (blue), $\mathrm{HD}$ (red), and $\mathrm{D}_{2}$ (green).

TABLE II. Polarizabilities, dipole moments, and masses of some selected molecules discussed in this work.

\begin{tabular}{lcccccc}
\hline \hline Molecule & $\begin{array}{c}\text { Mass } \\
(\mathrm{u})\end{array}$ & $\begin{array}{c}\text { Dipole moment } \\
\text { (D) }\end{array}$ & $\begin{array}{c}\text { Polarizability } \alpha_{\|} \\
\left(10^{-24} \mathrm{~cm}^{3}\right)\end{array}$ & $\begin{array}{c}\text { Polarizability } \alpha_{\perp} \\
\left(10^{-24} \mathrm{~cm}^{3}\right)\end{array}$ & $\begin{array}{c}\text { Mean polarizability/mass } \\
\left(10^{-24} \mathrm{~cm}^{3} \mathrm{u}^{-1}\right)\end{array}$ & Reference \\
\hline $\mathrm{OCS}$ & 60 & 0.71 & 1.14 & 0.75 & 0.0126 & 55 \\
$\mathrm{H}_{2}$ & 2 & 0 & 1.00220 & 0.70177 & 0.4510 & 56 \\
$\mathrm{HD}$ & 3 & 0 & 0.99483 & 0.69873 & 0.2987 & 56 \\
$\mathrm{D}_{2}$ & 4 & 0 & 0.98556 & 0.69484 & 0.2222 & 56 \\
$\mathrm{~N}_{2}$ & 28 & 0 & 2.2146 & 1.5188 & 0.0625 & 57 \\
$\mathrm{CH}_{4}$ & 16 & 0 & 2.4442 & 2.4442 & 0.1528 & 57 \\
$\mathrm{C}_{6} \mathrm{H}_{6}$ & 78 & 0 & 6.67 & 12.27 & 0.1094 & 58 \\
\hline \hline
\end{tabular}

\section{DISCUSSION}

The new HV-deflector combines several advantages of the designs that were used before. The deflector used by Wrede and Stern in the 1920s is distinguished by its simplicity (a wire parallel to a plain surface) but suffered in the resulting deflection force. ${ }^{5}$ The $a$-type deflector, as it was used by Chamberlain and Zorn, ${ }^{6}$ increased the force at a given electric potential. Nevertheless, it suffered from a deflection limit given by the molecules crashing into the rod. The group of Markus Arndt developed a deflector with a relatively large region of constant deflection force at the expense of the deflection strength. ${ }^{39}$ The deflector introduced here provides a strong deflection force combined with a large region where molecules can be deflected, although the force is not constant in this area. For a molecular beam with a small diameter, however, the force is in good approximation constant. The maximum achieved field strength in the $b$-type deflector was $363 \mathrm{kV} / \mathrm{cm}$. This is higher than the field strengths we typically achieve in an $a$ type deflector which are in the order of $220 \mathrm{kV} / \mathrm{cm}$. Moreover the highest field strength of the $a$-type deflector is located far outside the region of the molecular beam (see Fig. 2). For a $b$ type deflector, the molecules are deflected into the region of the highest field strength. The demonstrated degree of deflection was sufficient to completely separate a part of the molecular beam from the helium seed gas.

Both the measured degree of orientation and the simulated purity show an increase towards small laser positions for each specific skimmer position. This is attributed to the molecular beam being dispersed according to the molecules' effective dipole moments or, correspondingly, according to the specific rotational states. The field dressed state that correlates adiabatically with the field free rotational ground state of the molecule is most polar and therefore deflected the 
most. The ground state gives rise to the highest degree of orientation. This is reflected by the increased degree of orientation and purity in the deflected part of the molecular beam, which accounts for the larger contributions of low rotational quantum states. In addition, a decrease of the degree of orientation in regions where the excited rotational states remain (right-hand part of the specific molecular beam profiles) is observed. Figure 6 sketches the dispersion of the molecular beam passing the deflector in the cases of the deflector being switched off (a) and switched on (b). The movable skimmer selects a part of the beam (c), which subsequently disperses further due to the distinct transverse momenta of molecules in the different eigenstates. Therefore, a quantum state separation is obtained for each specific skimmer position.

The weighted mean of the purity provides a good measure for the purity of the unskimmed molecular beam. The region with the largest difference between the weighted mean purity and the unskimmed purity is the central part of the curves where the weighted mean is increased compared to the purity of the unskimmed molecular beam. This is attributed to the different shapes of the deflected ground state profile and the deflected profile of the rotationally excited states. The difference between the two profiles is small at low temperatures of our molecular beam, and, therefore, the weighted mean purity approximates to the real purity. The oscillations for the weighted mean purity in the central region is ascribed by the finite number of skimmer positions. We expect the weighted mean to underestimate the degree of orientation without a skimmer for the same reasons as discussed before for simulations of the ground state purity. A direct comparison of the measured degree of orientation and the simulated purity or quantum state distribution is, however, not possible due to the highly non-adiabatic orientation dynamics of the rotationally excited states. ${ }^{42}$ The character, and, therefore, the degree of orientation, of the specific quantum states was changed in the mixed field due to passage through real and avoided crossings while the laser pulse intensity was rising. Our simulations show that it should be possible to obtain a pure ground state sample for each specific skimmer position in the most deflected part of the molecular beam. Furthermore, from our simulations, we saw that the column density at this position is given by about $10 \%$ of the peak column density. This is also reflected in the measurements. The fluctuations in the degree of orientation in
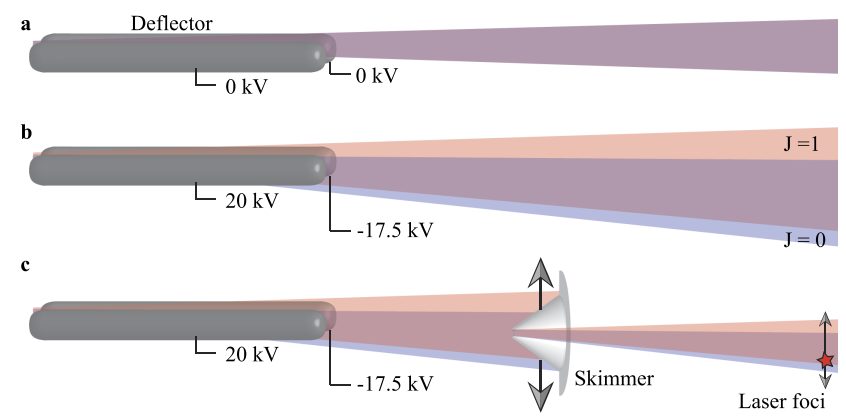

FIG. 6. Sketch of the dispersion of the molecular beam passing the deflector; the gray rods depict the deflector and the purple area the cross section of the molecular beam. (a) Deflector switched off. (b) Deflector switched on. (c) Influence of the skimmer. the most deflected part are attributed to the low statistics of these measurements.

The $b$-type deflector has the potential to separate nonpolar molecules. For the hydrogen isotopologues, we observed a linear increase of the effective dipole moment with increasing electric field strength. This is attributed to the small influence of the electric field on the field free rotational quantum states since all molecules in Table II are still in the perturbative regime for the simulated field strengths.

We observe in our simulation that the deflector can separate the different isotopologues, similar to previous conformerseparation experiments. ${ }^{14,16}$ However, the different rotational states of each isotopologue are not separable by the current deflector and molecular beam diameter and divergence. Thus a nuclear-spin purification, as previously demonstrated for water, ${ }^{60}$ is not possible with the current setup. This is due to the fact that the Stark effect in the currently available dc electric fields is dominated by the diagonal polarizability matrix element, i.e., essentially the scalar polarizability, whereas a significant influence of the off-diagonal matrix elements would render the states inequivalent. Our simulations show that a significant difference of effective dipole moments and, therefore, a separability of the $J=0$ and $J=1$ states would be obtained for a $25 \mu \mathrm{m}$ wide non-divergent molecular beam at the current maximum field strength of $363 \mathrm{kV} / \mathrm{cm}$.

Our simulations predict that spherical-top molecules such as methane $\left(\mathrm{CH}_{4}\right)$ are deflected fairly well, e.g., about $0.5 \mathrm{~mm}$ under the current experimental conditions. This might be beneficial for experiments where the molecules have to be separated from the seed gas. However, since for spherical-top molecules $\alpha_{\perp}=\alpha_{\|}$, their rotational quantum states cannot be separated due to their static polarizability at all.

\section{SUMMARY}

In conclusion, we have shown that the deflection of cold molecular beams with an inhomogeneous static electric field, given by the $b$-type-shaped deflector, enables the selection and a strong spatial separation of the most polar quantum states, i.e., the lowest-lying rotational states. Furthermore, our simulations showed that the deflector will allow the separation of isotopologues of non-polar molecules and that it enables background-gas free measurements of a whole new group of molecules. In comparison with the $a$-type deflector, it has the advantage that the highest field gradient is located close to the molecular beam position which results in a stronger deflection. In addition, due to the open structure of the deflector, strongly deflected molecules do not crash into the electrodes. This open structure also enables its utilization in merged beam experiments. ${ }^{61}$ Furthermore, a stronger deflection could be reached by optimizing the shape of the electrodes and the resulting field gradients. This strong deflection might be used to reduce the length of the deflector to increase the total molecular density at the region of interest.

The ability to disperse molecular beams by inhomogeneous electric fields is not limited to rotational state selection of small linear molecules. The new design makes a better separation of structural isomers of complex molecules as well as different sizes of individual clusters possible. ${ }^{9}$ These 
clean, well-defined, and spatially separated samples allow for novel experiments such as femtosecond pump-probe measurements, gas-phase $\mathrm{x}$-ray or electron diffraction, or tomographic reconstructions of molecular orbitals. In addition, it could be useful for isolating molecular signals in high-harmonic generation and attosecond experiments. Furthermore, state selection by deflection is highly advantageous in order to increase the degree of alignment and orientation to study complex molecules in the molecular frame..$^{11,62}$

\section{ACKNOWLEDGMENTS}

Besides DESY, this work has been supported by the Deutsche Forschungsgemeinschaft (DFG) through the excellence cluster "The Hamburg Center for Ultrafast ImagingStructure, Dynamics, and Control of Matter at the Atomic Scale" (CUI, EXC1074), the European Research Council through the Consolidator Grant COMOTION (NO. ERCKüpper-614507), and the Helmholtz Association "Initiative and Networking Fund."

${ }^{1}$ O. Stern, "Ein Weg zur experimentellen Prüfung der Richtungsquantelung im Magnetfeld," Z. Phys. 7, 249-253 (1921).

${ }^{2}$ W. Gerlach and O. Stern, "Der experimentelle Nachweis der Richtungsquantelung im Magnetfeld," Z. Phys. 9, 349-352 (1922).

${ }^{3}$ I. I. Rabi, S. Millman, P. Kusch, and J. R. Zacharias, "The molecular beam resonance method for measuring nuclear magnetic moments. The magnetic moments of ${ }_{3} L i^{6},{ }_{3} L i^{7}$ and ${ }_{9} F^{19}$," Phys. Rev. 55, 526-535 (1939).

${ }^{4}$ H. Kallmann and F. Reiche, "Über den Durchgang bewegter Moleküle durch inhomogene Kraftfelder,” Z. Phys. 6, 352-375 (1921).

${ }^{5}$ E. Wrede, "Über die Ablenkung von Molekularstrahlen elektrischer Dipolmoleküle im inhomogenen elektrischen Feld," Z. Phys. 44, 261-268 (1927).

${ }^{6}$ G. E. Chamberlain and J. C. Zorn, "Alkali polarizabilities by the atomic beam electrostatic deflection method," Phys. Rev. 129, 677 (1963).

${ }^{7}$ F. Filsinger, J. Küpper, G. Meijer, L. Holmegaard, J. H. Nielsen, I. Nevo, J. L. Hansen, and H. Stapelfeldt, "Quantum-state selection, alignment, and orientation of large molecules using static electric and laser fields," J. Chem. Phys. 131, 064309 (2009); e-print arXiv:0903.5413 [physics].

${ }^{8}$ A. J. de Nijs and H. L. Bethlem, "On deflection fields, weak-focusing and strong-focusing storage rings for polar molecules," Phys. Chem. Chem. Phys. 13, 19052-19058 (2011); e-print arXiv:1105.1913 [physics].

${ }^{9}$ Y.-P. Chang, D. A. Horke, S. Trippel, and J. Küpper, "Spatially-controlled complex molecules and their applications," Int. Rev. Phys. Chem. 34, 557590 (2015); e-print arXiv:1505.05632 [physics].

${ }^{10}$ N. F. Ramsey, Molecular Beams, The International Series of Monographs on Physics (Oxford University Press, London, GB, 1956), reprinted in Oxford Classic Texts in the Physical Sciences, 2005.

${ }^{11}$ L. Holmegaard, J. H. Nielsen, I. Nevo, H. Stapelfeldt, F. Filsinger, J. Küpper, and G. Meijer, "Laser-induced alignment and orientation of quantum-stateselected large molecules," Phys. Rev. Lett. 102, 023001 (2009); e-print arXiv:0810.2307 [physics].

${ }^{12}$ K. Wohlfart, F. Grätz, F. Filsinger, H. Haak, G. Meijer, and J. Küpper, "Alternating-gradient focusing and deceleration of large molecules," Phys. Rev. A 77, 031404(R) (2008).

${ }^{13}$ S. Putzke, F. Filsinger, H. Haak, J. Küpper, and G. Meijer, "Rotationalstate-specific guiding of large molecules," Phys. Chem. Chem. Phys. 13, 18962 (2011); e-print arXiv:1103.5080 [physics].

${ }^{14}$ F. Filsinger, J. Küpper, G. Meijer, J. L. Hansen, J. Maurer, J. H. Nielsen, L. Holmegaard, and H. Stapelfeldt, "Pure samples of individual conformers: The separation of stereo-isomers of complex molecules using electric fields," Angew. Chem., Int. Ed. 48, 6900-6902 (2009).

${ }^{15}$ F. Filsinger, U. Erlekam, G. von Helden, J. Küpper, and G. Meijer, "Selector for structural isomers of neutral molecules," Phys. Rev. Lett. 100, 133003 (2008); e-print arXiv:0802.2795 [physics].

${ }^{16}$ T. Kierspel, D. A. Horke, Y.-P. Chang, and J. Küpper, "Spatially separated polar samples of the cis and trans conformers of 3-fluorophenol," Chem. Phys. Lett. 591, 130-132 (2014); e-print arXiv:1312.4417 [physics].
${ }^{17}$ S. Trippel, Y.-P. Chang, S. Stern, T. Mullins, L. Holmegaard, and J. Küpper, "Spatial separation of state- and size-selected neutral clusters," Phys. Rev. A 86, 033202 (2012); e-print arXiv:1208.4935 [physics].

${ }^{18}$ J. Küpper, S. Stern, L. Holmegaard, F. Filsinger, A. Rouzée, A. Rudenko, P. Johnsson, A. V. Martin, M. Adolph, A. Aquila, S. Bajt, A. Barty, C. Bostedt, J. Bozek, C. Caleman, R. Coffee, N. Coppola, T. Delmas, S. Epp, B. Erk, L. Foucar, T. Gorkhover, L. Gumprecht, A. Hartmann, R. Hartmann, G. Hauser, P. Holl, A. Hömke, N. Kimmel, F. Krasniqi, K.-U. Kühnel, J. Maurer, M. Messerschmidt, R. Moshammer, C. Reich, B. Rudek, R. Santra, I. Schlichting, C. Schmidt, S. Schorb, J. Schulz, H. Soltau, J. C. H. Spence, D. Starodub, L. Strüder, J. Thøgersen, M. J. J. Vrakking, G. Weidenspointner, T. A. White, C. Wunderer, G. Meijer, J. Ullrich, H. Stapelfeldt, D. Rolles, and H. N. Chapman, "X-ray diffraction from isolated and strongly aligned gas-phase molecules with a free-electron laser," Phys. Rev. Lett. 112, 083002 (2014); e-print arXiv:1307.4577 [physics].

${ }^{19}$ F. Filsinger, G. Meijer, H. Stapelfeldt, H. Chapman, and J. Küpper, "Stateand conformer-selected beams of aligned and oriented molecules for ultrafast diffraction studies," Phys. Chem. Chem. Phys. 13, 2076-2087 (2011); e-print arXiv: 1009.0871 [physics].

${ }^{20}$ A. Barty, J. Küpper, and H. N. Chapman, "Molecular imaging using x-ray free-electron lasers," Annu. Rev. Phys. Chem. 64, 415-435 (2013).

${ }^{21}$ N. L. M. Müller, "Electron diffraction and controlled molecules," Dissertation, Dr. rer. nat. (Universität Hamburg, Hamburg, Germany, 2016).

${ }^{22}$ S. Y. T. van de Meerakker, H. L. Bethlem, and G. Meijer, "Taming molecular beams," Nat. Phys. 4, 595 (2008).

${ }^{23}$ M. Schnell and G. Meijer, "Cold molecules: Preparation, applications, and challenges," Angew. Chem., Int. Ed. 48, 6010-6031 (2009).

${ }^{24}$ M. T. Bell and T. P. Softley, "Ultracold molecules and ultracold chemistry," Mol. Phys. 107, 99-132 (2009).

${ }^{25}$ S. D. Hogan, M. Motsch, and F. Merkt, "Deceleration of supersonic beams using inhomogeneous electric and magnetic fields," Phys. Chem. Chem. Phys. 13, 18705-18723 (2011).

${ }^{26}$ S. Y. T. van de Meerakker, H. L. Bethlem, N. Vanhaecke, and G. Meijer, "Manipulation and control of molecular beams," Chem. Rev. 112, 48284878 (2012).

${ }^{27}$ H. L. Bethlem, M. R. Tarbutt, J. Küpper, D. Carty, K. Wohlfart, E. A. Hinds, and G. Meijer, "Alternating gradient focusing and deceleration of polar molecules," J. Phys. B 39, R263-R291 (2006); e-print arXiv:0604020 [physics].

${ }^{28}$ S. Putzke, F. Filsinger, J. Küpper, and G. Meijer, "Alternating-gradient focusing of the benzonitrile-argon van der Waals complex," J. Chem. Phys. 137, 104310 (2012); e-print arXiv:1208.3188 [physics].

${ }^{29}$ B. C. Bohrer, S. I. Merenbloom, S. L. Koeniger, A. E. Hilderbrand, and D. E. Clemmer, "Biomolecule analysis by ion mobility spectrometry," Annu. Rev. Anal. Chem. 1, 293-327 (2008).

${ }^{30}$ T. Wyttenbach, N. A. Pierson, D. E. Clemmer, and M. T. Bowers, "Ion mobility analysis of molecular dynamics," Annu. Rev. Phys. Chem. 65, 175-196 (2014).

${ }^{31}$ H. Stapelfeldt, H. Sakai, E. Constant, and P. B. Corkum, "Deflection of neutral molecules using the nonresonant dipole force," Phys. Rev. Lett. 79, 2787-2790 (1997).

${ }^{32}$ B. S. Zhao, H. S. Chung, K. Cho, S. H. Lee, S. Hwang, J. Yu, Y. H. Ahn, J. Y. Sohn, D. S. Kim, W. K. Kang, and D. S. Chung, "Molecular lens of the nonresonant dipole force," Phys. Rev. Lett. 85, 2705-2708 (2000).

${ }^{33}$ R. Fulton, A. I. Bishop, and P. F. Barker, "Optical stark decelerator for molecules," Phys. Rev. Lett. 93, 243004 (2004).

${ }^{34}$ X. N. Sun, L. Y. Kim, B. S. Zhao, and D. S. Chung, "Rotational-statedependent dispersion of molecules by pulsed optical standing waves," Phys. Rev. Lett. 115, 223001 (2015).

${ }^{35}$ S. R. Procter, Y. Yamakita, F. Merkt, and T. P. Softley, "Controlling the motion of hydrogen molecules," Chem. Phys. Lett. 372, 667-675 (2003).

${ }^{36}$ H. Scheffers and J. Stark, "Einfluß des elektrischen Feldes auf Alkaliatome im Atomstrahlversuch," Phys. Z 35, 625-627 (1934).

${ }^{37}$ E. Gershnabel and I. S. Averbukh, "Deflection of field-free aligned molecules," Phys. Rev. Lett. 104, 153001 (2010).

${ }^{38}$ E. Gershnabel and I. S. Averbukh, "Deflection of rotating symmetric top molecules by inhomogeneous fields," J. Chem. Phys. 135, 084307 (2011).

${ }^{39}$ A. Stefanov, M. Berninger, and M. Arndt, "A novel design for electric field deflectometry on extended molecular beams," Meas. Sci. Technol. 19, 055801 (2008).

${ }^{40} \mathrm{~S}$. Eibenberger, S. Gerlich, M. Arndt, J. Tüxen, and M. Mayor, "Electric moments in molecule interferometry," New J. Phys. 13, 043033 (2011). 
${ }^{41}$ J. H. Nielsen, H. Stapelfeldt, J. Küpper, B. Friedrich, J. J. Omiste, and R. González-Férez, "Making the best of mixed-field orientation of polar molecules: A recipe for achieving adiabatic dynamics in an electrostatic field combined with laser pulses," Phys. Rev. Lett. 108, 193001 (2012); e-print arXiv:1204.2685 [physics].

${ }^{42}$ J. S. Kienitz, S. Trippel, T. Mullins, K. Długołęcki, R. González-Férez, and J. Küpper, "Adiabatic mixed-field orientation of ground-state-selected carbonyl sulfide molecules," Chem. Phys. Chem. 17, 3740-3746 (2016); e-print arXiv:1607.05615 [physics].

${ }^{43}$ W. Gordy and R. L. Cook, Microwave Molecular Spectra, 3rd ed. (John Wiley \& Sons, New York, NY, USA, 1984).

${ }^{44}$ Y.-P. Chang, F. Filsinger, B. Sartakov, and J. Küpper, "CMIstark: Python package for the stark-effect calculation and symmetry classification of linear, symmetric and asymmetric top wavefunctions in dc electric fields," Comput. Phys. Comm. 185, 339-349 (2014); e-print arXiv:1308.4076 [physics].

${ }^{45}$ U. Even, "Pulsed supersonic beams from high pressure source: Simulation results and experimental measurements," Adv. Chem. 2014, 636042.

${ }^{46}$ R. V. Latham, High Voltage Vaccum Insulation: The Physical Basis (Academic Press, London, 1981).

${ }^{47}$ D. Auerbach, E. E. A. Bromberg, and L. Wharton, "Alternate-gradient focusing of molecular beams," J. Chem. Phys. 45, 2160 (1966).

${ }^{48}$ J. G. Kalnins, G. Lambertson, and H. Gould, "Improved alternating gradient transport and focusing of neutral molecules," Rev. Sci. Instrum. 73, 25572565 (2002).

${ }^{49}$ J. S. Kienitz, "Orientation of state selected OCS molecules in mixed strong dc and laser fields," Dissertation, Dr. rer. nat. (Universität Hamburg, Hamburg, Germany, 2016).

${ }^{50}$ U. Even, J. Jortner, D. Noy, N. Lavie, and N. Cossart-Magos, "Cooling of large molecules below $1 \mathrm{~K}$ and He clusters formation," J. Chem. Phys. 112, 8068-8071 (2000).

${ }^{51}$ S. Trippel, T. Mullins, N. L. M. Müller, J. S. Kienitz, K. . Długołęcki, and J. Küpper, "Strongly aligned and oriented molecular samples at a kHz repetition rate," Mol. Phys. 111, 1738 (2013); e-print arXiv:1301.1826 [physics.atom-ph].
${ }^{52}$ J. H. Nielsen, P. Simesen, C. Z. Bisgaard, H. Stapelfeldt, F. Filsinger, B. Friedrich, G. Meijer, and J. Küpper, "Stark-selected beam of ground-state OCS molecules characterized by revivals of impulsive alignment," Phys. Chem. Chem. Phys. 13, 18971-18975 (2011); e-print arXiv:1105.2413 [physics].

${ }^{53}$ R. H. Orcutt, "Influence of molecular quadrupole moments on the second virial coefficient," J. Chem. Phys. 39, 605-608 (1963).

${ }^{54}$ H.-O. Hamaguchi, A. Buckingham, and W. Jones, "Determination of derivatives of the polarizability anisotropy in diatomic molecules," Mol. Phys. 43, 1311-1319 (1981).

${ }^{55}$ G. R. Alms, A. Burnham, and W. H. Flygare, "Measurement of the dispersion in polarizability anisotropies," J. Chem. Phys. 63, 3321-3326 (1975).

${ }^{56} \mathrm{~J}$. Rychlewski, "An accurate calculation of the polarizability of the hydrogen molecule and its dependence on rotation, vibration and isotopic substitution," Mol. Phys. 41, 833-842 (1980).

${ }^{57}$ M. A. Buldakov, V. N. Cherepanov, Y. N. Kalugina, N. Zvereva-Loëte, and V. Boudon, "Static polarizability surfaces of the van der Waals complex $\mathrm{CH}_{4}-\mathrm{N}_{2}$," J. Chem. Phys. 132, 164304 (2010).

${ }^{58}$ N. J. Bridge and A. D. Buckingham, "The polarization of laser light scattered by gases," Proc. R. Soc. A 295, 334-349 (1966).

${ }^{59} \mathrm{~K}$. Wohlfart, "Alternating-gradient focusing and deceleration of large molecules," Dissertation, Dr. rer. nat. (Free University, Berlin, Germany, 2008).

${ }^{60}$ D. A. Horke, Y.-P. Chang, K. Długołęcki, and J. Küpper, "Separating para and ortho water," Angew. Chem., Int. Ed. 53, 11965-11968 (2014); e-print arXiv:1407.2056 [physics].

${ }^{61}$ A. B. Henson, S. Gersten, Y. Shagam, J. Narevicius, and E. Narevicius, "Observation of resonances in penning ionization reactions at sub-kelvin temperatures in merged beams," Science 338, 234-238 (2012).

${ }^{62}$ L. Holmegaard, J. L. Hansen, L. Kalhøj, S. L. Kragh, H. Stapelfeldt, F. Filsinger, J. Küpper, G. Meijer, D. Dimitrovski, M. Abu-samha, C. P. J. Martiny, and L. B. Madsen, "Photoelectron angular distributions from strong-field ionization of oriented molecules," Nat. Phys. 6, 428 (2010); e-print arXiv:1003.4634 [physics]. 